\title{
Clinical characteristics of 116 hospitalized patients with COVID-19 in Wuhan, China: a single-centered, retrospective, observational study
}

Shiqiang Xiong ${ }^{1 \dagger}$, Lin Liu ${ }^{2 \dagger}$, Feng Lin ${ }^{1}$, Jinhu Shi ${ }^{1}$, Lei Han ${ }^{3}$, Huijian Liư', Lewei He${ }^{1}$, Qijun Jiang ${ }^{1}$, Zeyang Wang ${ }^{1}$, Wenbo Fu', Zhigang $\mathrm{Li}^{1}$, Qing Lu', Zhinan Chen ${ }^{1}$ and Shifang Ding ${ }^{1 *}$ (D)

\begin{abstract}
Background: A cluster of acute respiratory illness, now known as Corona Virus Disease 2019 (COVID-19) caused by 2019 novel coronavirus (SARS-CoV-2), has become a global pandemic. Aged population with cardiovascular diseases are more likely be to infected with SARS-CoV-2 and result in more severe outcomes and elevated case-fatality rate. Meanwhile, cardiovascular diseases have a high prevalence in the middle-aged and elderly population. However, despite of several researches in COVID-19, cardiovascular implications related to it still remains largely unclear. Therefore, a specific analysis in regard to cardiovascular implications of COVID-19 patients is in great need.

Methods: In this single-centered, retrospective, observational study, 116 patients with laboratory-confirmed COVID19 were enrolled, who admitted to the General Hospital of Central Theater Command (Wuhan, China) from January 20 to March 8, 2020. The demographic data, underlying comorbidities, clinical symptoms and signs, laboratory findings, chest computed tomography, treatment measures, and outcome data were collected from electronic medical records. Data were compared between non-severe and severe cases.

(Continued on next page)
\end{abstract}

\footnotetext{
* Correspondence: dsfmdwh@163.com

${ }^{\dagger}$ Shiqiang Xiong and Lin Liu contributed equally to this work.

'Department of Cardiology, the General Hospital of Central Theater Command, Wuhan 430000, China

Full list of author information is available at the end of the article
}

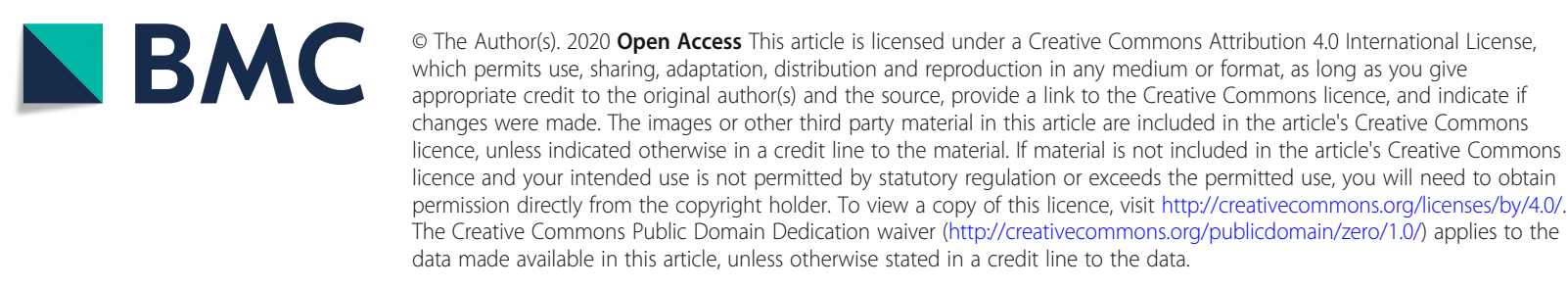


(Continued from previous page)

Results: Of 116 hospitalized patients with COVID-19, the median age was 58.5 years (IQR, 47.0-69.0), and 36 (31.0\%) were female. Hypertension (45 [38.8\%]), diabetes (19 [16.4\%]), and coronary heart disease (17 [14.7\%]) were the most common coexisting conditions. Common symptoms included fever [99 (85.3\%)], dry cough (61 [52.6\%]), fatigue (60 [51.7\%]), dyspnea (52 [44.8\%]), anorexia (50 [43.1\%]), and chest discomfort (50 [43.1\%]). Local and/or bilateral patchy shadowing were the typical radiological findings on chest computed tomography. Lymphopenia (lymphocyte count, $1.0 \times 10^{9} / \mathrm{L}$ [IQR, 0.7-1.3]) was observed in 66 patients (56.9\%), and elevated lactate dehydrogenase (245.5 U/L [IQR, 194.3-319.8]) in 69 patients (59.5\%). Hypokalemia occurred in 24 (20.7\%) patients. Compared with non-severe cases, severe cases were older (64.0 years [IQR, 53.0-76.0] vs 56.0 years [IQR, 37.0-64.0]), more likely to have comorbidities (35 [63.6\%] vs 24 [39.3\%]), and more likely to develop acute cardiac injury (19 [34.5\%] vs 4 [6.6\%]), acute heart failure (18 [32.7\%] vs 3 [4.9\%]), and ARDS (20 [36.4\%] vs 0 [0\%]). During hospitalization, the prevalence of new onset hypertension was significantly higher in severe patients (55.2\% vs 19.0\%) than in non-severe ones.

Conclusions: In this single-centered, retrospective, observational study, we found that the infection of SARS-CoV-2 was more likely to occur in middle and aged population with cardiovascular comorbidities. Cardiovascular complications, including new onset hypertension and heart injury were common in severe patients with COVID-19. More detailed researches in cardiovascular involvement in COVID-19 are urgently needed to further understand the disease.

Keywords: SARS-CoV-2, COVID-19, Cardiovascular disease, Hypertension, Coronary heart disease

\section{Background}

In early December 2019, a cluster of acute respiratory illness, now known as Corona Virus Disease 2019 (COVID19) caused by 2019 novel coronavirus (SARS-CoV-2), occurred in Wuhan, Hubei Province, China [1-4]. COVID19 has rapidly spread all over the world and become a global pandemic. Although several recent studies have described the clinical spectrum of COVID-19, including general epidemiological characteristics, clinical manifestations, and clinical outcomes of patients [4-7], cardiovascular implications of COVID-19 remain largely unclear.

Cardiovascular diseases have a high prevalence in middle-aged and elderly population [8]. Aged population with comorbidities, such as cardiovascular diseases are more susceptible to COVID-19 and result in severe outcomes and elevated case-fatality rate $[5,9]$. Acute cardiac injury is one of the common complications in COVID-19 patients [7]. These findings suggest that cardiovascular system is tightly implicated in COVID-19. By collecting data from 116 laboratory-confirmed cases who were admitted to the General Hospital of Central Theater Command, we sought to provide an up-to-date description of the clinical characteristics and cardiovascular status of patients with COVID-19. This study would not only identify the clinical manifestations with greater precision, but also provide assistance for non-cardiovascular specialists to manage COVID-19 patients with cardiovascular disorders.

\section{Methods}

\section{Study design and participants}

For this single-centered, retrospective, observational study, we recruited patients with laboratory-confirmed
COVID-19 admitted to the General Hospital of Central Theater Command (Wuhan, China) from January 20 to March 8, 2020. A confirmed case with SARS-CoV-2 infection was defined as a positive result for real-time reversetranscriptase polymerase-chain-reaction (RT-PCR) assay for pharyngeal swab specimens. The General Hospital of Central Theater Command is one of the major hospitals responsible for COVID-19 treatment designated by the government. At the time of admission, the severity of COVID-19 was defined as mild, moderate, severe and critical cases based on the 6th edition guideline issued by the National Health Commission of China. Mild patients were not admitted in this designated hospital. Moderate cases were having mild symptoms of respiratory infections with pneumonia. Severe cases were defined as dyspnea, respiratory frequency $\geq 30 / \mathrm{min}$, blood oxygen saturation $\leq 93 \%$, $\mathrm{PaO} 2 / \mathrm{FiO} 2$ ratio $\leq 300 \mathrm{mmHg}$, and/or pulmonary inflammation progressing $>50 \%$ within 24 to $48 \mathrm{~h}$. Critical cases were those who exhibited respiratory failure, shock, and/ or multiple organ dysfunction. In this study, moderate cases were categorized as the non-severe group; severe and critical cases were categorized as the severe group. Oral informed consent was obtained from patients. All the protocol in this study was approved by the Ethics Commission of the General Hospital of Central Theater Command ([2020]025-1). The clinical outcomes (ie, discharge, mortality, and length of stay) were monitored up to April 13,2020 , the final date of follow-up.

\section{Data collection}

We obtained the demographic data, medical history, underlying comorbidities, clinical symptoms and signs, 
laboratory findings, chest computed tomography (CT), treatment measures, and outcome data from electronic medical records for all hospitalized patients with laboratory confirmed COVID-19. ARDS and shock were defined in accordance with the guidance of WHO for COVID-19 [10]. The diagnosis of acute kidney injury was based on the highest serum creatinine level and urine output [11]. Cardiac injury was defined if the serum concentration of hypersensitive cardiac troponin $\mathrm{T}(\mathrm{c} \operatorname{TnT})$ was above the upper limit of the reference range $(>0.02 \mathrm{ng} / \mathrm{mL})$. Acute heart failure was defined based on the typical symptoms that may be accompanied by signs caused by a structural and/or functional cardiac abnormality [12]. Hypertension is defined in adults as the results of systolic blood pressure $\geq 140 \mathrm{mmHg}$ and/ or diastolic blood pressure $\geq 90 \mathrm{mmHg}$ three times on different days. History of coronary heart disease was defined as having any of the following: evidence of myocardial infarction on the baseline electrocardiograph, selfreport of a prior history of a cardiac procedure (coronary artery bypass surgery, coronary angioplasty, balloon angioplasty, atherectomy, stent, percutaneous transluminal coronary angioplasty, or percutaneous coronary intervention), self-reported history of myocardial infarction, or angina with $\geq 50 \%$ angiographic obstruction of a major coronary artery. The information was validated by hospital records.

\section{Statistical analysis}

Continuous variables were presented as means and standard error (SEM) or medians and interquartile ranges (IQR) values as appropriate. Categorical variables were expressed as the counts and percentages in each category. Independent group t-test was used for continuous variable, when the data conformed to normal distribution; otherwise, the Mann-Whitney test was applied. Data (not normal distributed) from repeated measures were compared using the generalized linear mixed model. The chi-square test and Fisher's exact test were applied for categorical variables as appropriate. Twosided $p$-values less than 0.05 were considered statistically significant. All analyses were conducted with GraphPad Prism software (version 8.0).

\section{Results}

\section{Baseline characteristics}

The study population included 116 hospitalized patients with laboratory-confirmed COVID-19 (Table 1). The median age was 58.5 years (IQR, 47.0-69.0), and 36 $(31.0 \%)$ were females. Fifty-nine $(50.9 \%)$ patients had at least one underlying disorder. Hypertension (45 [38.8\%]), diabetes (19 [16.4\%]), coronary heart disease (17 [14.7\%]), and cerebrovascular diseases (8 [6.9\%]) were the most common coexisting conditions (Table 1). Fever
(85.3\%), dry cough $(52.6 \%)$, fatigue $(51.7 \%)$, anorexia (43.1\%), dyspnea (44.8\%), and chest discomfort (43.1\%) were the most common symptoms, whereas dizziness (6.0\%), nasal obstruction (5.2\%), abdominal pain (2.6\%), hemoptysis $(0.9 \%)$ were less common (Table 1$)$. The median durations from first symptoms to dyspnea and hospital admission were 4.5 days (IQR, $0-9.0$ ), and 8.0 days (IQR, 4.0-11.0) respectively (Table 1 ).

On admission, 61 and 55 patients were categorized into non-severe and severe subgroups, respectively. The age differed significantly between the two groups (median age, non-severe vs severe, 56.0 years [IQR, 37.064.0] vs 64.0 years [IQR, 53.0-76.0]; $P<0.001$ ). Severe cases were more prone to having underlying comorbidities, including coronary heart disease $(13$ [23.6\%] vs 4 [6.6\%], cerebrovascular diseases $(7[12.7 \%]$ vs $1[1.6 \%])$, and malignancy $(4[7.3 \%]$ vs $0[0.0 \%])$. Compared with the non- severe group, dyspnea and fatigue were more frequently reported in severe patients. Vital signs were recorded on the day of admission to hospital for all patients. Respiratory rate was higher in severe cases as compared with non-severe cases (20.0 [IQR, 18.0-25.0] vs 19.0 [IQR, 18.0-20.0; $P=0.030]$ ). While, heart rate, mean arterial pressure, and body temperature showed no significant difference between the two groups (all $P>0.05)$.

\section{Laboratory and radiologic findings at presentation}

The most common pattern on chest CT was bilateral patchy shadowing (69.9\%). These imaging alterations were more prominent in severe patients (47 [85.5\%] vs 36 [59.0\%]; $P=0.002$ ) (Table 2).

There were numerous differences in laboratory findings between severe and non-severe cases (Table 3). Laboratory abnormalities were more obviously seen in severe cases, including lower counts of lymphocyte, $\mathrm{T}$ cells, $\mathrm{CD} 4^{+}$and $\mathrm{CD} 8^{+} \mathrm{T}$ cells, and elevated levels of neutrophil count, procalcitonin, c-reactive protein, interleukin 6, D-dimer, creatinine, blood urea nitrogen, lactate dehydrogenase, myoglobin, cTnT, and NT-proBNP (all $P<0.05)$. Hypokalemia $(20.7 \%)$ was prevailing in both severe and non-severe patients, though no statistical difference was found between them.

\section{Complications, main interventions, and outcomes}

The organ dysfunction and treatment of the 116 patients were shown in Table 4. On April 21, 2020, a total of 109 patients (94.0\%) had been discharged, and 7 patients (6.0\%) had died. Among the 116 patients, the most common complication was acute cardiac injury (23 [19.8\%]), followed by acute heart failure (21 [18.1\%]), ARDS (20 [17.2\%]), shock (16 [13.8\%]), or liver dysfunction (15 [12.9\%]). Severe cases were more liable to having one of these complications than non- severe cases. 
Table 1 Baseline Characteristics of Patients Infected With COVID-19

\begin{tabular}{|c|c|c|c|c|}
\hline & \multicolumn{3}{|l|}{ Disease severity } & \multirow[t]{2}{*}{ PValue } \\
\hline & Total $(N=116)$ & Non-severe $(n=61)$ & Severe $(n=55)$ & \\
\hline Age, median (IQR), y & $58.5(47.0-69.0)$ & $56.0(37.0-64.0)$ & $64.0(53.0-76.0)$ & $<0.001$ \\
\hline $0-14$ y & 0 & 0 & 0 & - \\
\hline $15-49 y$ & $33 / 116(28.4)$ & 24/61 (39.3) & $9 / 55(16.4)$ & - \\
\hline $50-64$ y & $41 / 116(35.3)$ & 23/61 (37.7) & 18/55 (32.7) & - \\
\hline$\geq 65 y$ & $42 / 116(36.2)$ & $14 / 61(23.0)$ & 28/55 (50.9) & - \\
\hline \multicolumn{5}{|l|}{ Sex -No., \% } \\
\hline Female & $36 / 116(31.0)$ & 19/61 (31.1) & 17/55 (30.9) & \multirow[t]{2}{*}{1.000} \\
\hline Male & $80 / 116(69.0)$ & 42/61 (68.9) & $38 / 55(69.1)$ & \\
\hline \multicolumn{5}{|l|}{ Comorbidities -No., \% } \\
\hline Any & $59 / 116(50.9)$ & 24/61 (39.3) & $35 / 55(63.6)$ & 0.009 \\
\hline COPD & $1 / 116(0.9)$ & 0/61 (0.0) & $1 / 55(1.8)$ & 0.290 \\
\hline Diabetes & 19/116 (16.4) & $8 / 61(13.1)$ & $11 / 55(20.0)$ & 0.317 \\
\hline Hypertension & 45/116 (38.8) & 19/61 (31.1) & $26 / 55(47.3)$ & 0.075 \\
\hline Coronary heart disease & $17 / 116(14.7)$ & 4/61 (6.6) & 13/55 (23.6) & 0.009 \\
\hline Cerebrovascular disease & $8 / 116(6.9)$ & $1 / 61(1.6)$ & $7 / 55(12.7)$ & 0.019 \\
\hline Malignancy & $4 / 116(3.4)$ & 0/61 (0.0) & 4/55 (7.3) & 0.032 \\
\hline Chronic kidney disease & $0 / 116(0.0)$ & 0/61 (0.0) & 0/55 (0.0) & - \\
\hline Chronic liver disease & $2 / 116(1.7)$ & $1 / 61(1.6)$ & $1 / 55(1.8)$ & 0.941 \\
\hline HIV infection & $0 / 116(0.0)$ & $0 / 61(0.0)$ & 0/55 (0.0) & - \\
\hline \multicolumn{5}{|c|}{ Signs and symptoms-No., \% } \\
\hline Fever & $99 / 116(85.3)$ & 49/61 (80.3) & $50 / 55(91.0)$ & 0.108 \\
\hline Fatigue & $60 / 116(51.7)$ & $26 / 61(42.6)$ & $34 / 55(61.9)$ & 0.039 \\
\hline Dry cough & $61 / 116(52.6)$ & $31 / 61(50.8)$ & $30 / 55(54.5)$ & 0.688 \\
\hline Anorexia & $50 / 116(43.1)$ & $27 / 61(44.3)$ & 23/55 (41.8) & 0.791 \\
\hline Myalgia & $32 / 116(27.6)$ & 19/61 (31.1) & 13/55 (23.6) & 0.366 \\
\hline Dyspnea & $52 / 116(44.8)$ & 21/61 (34.4) & $31 / 55(56.4)$ & 0.018 \\
\hline Expectoration & $31 / 116(26.7)$ & $17 / 61(27.9)$ & $14 / 55(25.5)$ & 0.769 \\
\hline Hemoptysis & $1 / 116(0.9)$ & 0/61 (0.0) & $1 / 55(1.8)$ & 0.290 \\
\hline Pharyngalgia & 15/116 (12.9) & $7 / 61(11.5)$ & $8 / 55(14.5)$ & 0.623 \\
\hline Nasal obstruction & $6 / 116(5.2)$ & 4/61 (6.6) & 2/55 (3.6) & 0.478 \\
\hline Diarrhea & $17 / 116(14.7)$ & $14 / 61(23.0)$ & $3 / 55(5.5)$ & 0.008 \\
\hline Nausea & 10/116 (8.6) & 4/61 (6.6) & $6 / 55(11.0)$ & 0.404 \\
\hline Dizziness & $7 / 116(6.0)$ & 2/61 (3.3) & $5 / 55(9.1)$ & 0.189 \\
\hline Headache & $6 / 116(5.2)$ & $5 / 61(8.2)$ & $1 / 55(1.8)$ & 0.121 \\
\hline Vomiting & $5 / 116(4.3)$ & $3 / 61(4.9)$ & 2/55 (3.6) & 0.734 \\
\hline Chill & $24 / 116(20.7)$ & 13/61 (21.3) & $11 / 55(20.0)$ & 0.901 \\
\hline Shiver & $5 / 116(4.3)$ & $3 / 61(4.9)$ & 2/55 (3.6) & 0.734 \\
\hline Abdominal pain & $3 / 116(2.6)$ & 3/61 (4.9) & $0 / 55(0.0)$ & 0.096 \\
\hline Chest discomfort & $50 / 116(43.1)$ & 22/61 (36.1) & 28/55 (51.0) & 0.107 \\
\hline Palpitation & $13 / 116(11.2)$ & $7 / 61(11.5)$ & $6 / 55(11.0)$ & 0.923 \\
\hline Sleep disorders & 27/116 (23.3) & 15/61 (24.6) & $12 / 55(21.8)$ & 0.724 \\
\hline \multicolumn{5}{|c|}{ Onset of symptom to, median (IQR), d } \\
\hline Hospital admission & $8.0(4.0-11.0)$ & $8.0(4.0-11.0)$ & $8.0(4.0-11.0)$ & 0.910 \\
\hline
\end{tabular}


Table 1 Baseline Characteristics of Patients Infected With COVID-19 (Continued)

\begin{tabular}{|c|c|c|c|c|}
\hline & \multicolumn{3}{|l|}{ Disease severity } & \multirow[t]{2}{*}{ PValue } \\
\hline & Total $(N=116)$ & Non-severe $(n=61)$ & Severe $(n=55)$ & \\
\hline Dyspnea & $4.5(0.0-9.0)$ & $3.0(0.0-12.0)$ & $6.0(1.5-8.5)$ & 0.649 \\
\hline Heart rate on admission, median (IQR), bpm & $86.0(80.0-98.0)$ & $85.5(80.0-97.5)$ & $86.0(80.0-98.0)$ & 0.958 \\
\hline Respiratory rate on admission, median (IQR) & $19.0(18.0-23.0)$ & $19.0(18.0-20.0)$ & $20.0(18.0-25.0)$ & 0.030 \\
\hline Mean arterial pressure on admission, median (IQR), mmHg & $96.7(86.7-103.3)$ & $96.7(86.7-103.5)$ & $96.7(86.7-103.3)$ & 1.000 \\
\hline Temperature on admission, median (IQR), ${ }^{\circ} \mathrm{C}$ & $36.8(36.5-37.5)$ & $36.7(36.5-37.3)$ & $37.0(36.4-37.8)$ & 0.138 \\
\hline
\end{tabular}

All patients were treated in isolation. 112 (96.6\%) patients received antiviral treatment, including oseltamivir phosphatecas $(91,[78.4 \%])$, lopinave/litonawe (74, [63.8\%]), arbidol hydrochloride (27, [21.4\%]), and ribavirin Injection (71, [61.2\%]). Antibacterial therapy was performed for most patients (113, [97.4\%]). Twelve (10.3\%) patients adopting antifungal medications were all enrolled in severe group. Systemic corticosteroid was given to $47.4 \%$ of cases and more so in the severe patients than in the non-severe group $(74.5 \%$ vs $23.0 \%, P<0.0001)$. Obviously, more severe cases received mechanical ventilation (non-invasive: $32.7 \%$ vs. $0 \%, P<0.0001$; invasive: $18.2 \%$ vs. $0 \%, P<0.0001)$ as compared with non-severe cases. Three severe patients were treated with extracorporeal membrane oxygenation. Traditional Chinese medical herbal treatment and immune support therapy were applied in most cases. Overall, all these interventions were initiated in significantly higher percentages of severe patients.

Of the 7 deceased patients, all patients developed ARDS, 4 had sepsis or sepsis shock, 1 had gastrointestinal and cerebral hemorrhage. As the disease progressed and clinical status deteriorated, the levels of D-dimer, creatinine, blood urea, lactate dehydrogenase, and cTnT progressively increased before death. Among the dead patients, all received antiviral therapy, antibacterial therapy, and immune support therapy (use of intravenous immunoglobin and/or thymopeptides injection). Based on the 6th edition guideline issued by the National Health Commission of China, Hydroxychloroquine had not been recommend in the treatment of COVID-19 yet. Meanwhile, traditional Chinese medical herbal treatment, including Lianhua Qingwen Capsule and oral use of Chinese herbal decoction, like Lung cleansing and detoxification soup, were recommended and applied in most hospitalized patients. This part of treatment was managed by the doctor majoring in traditional Chinese medicine. In the present study, 5 non-survivors received Lianhua Qingwen Capsule therapy.

\section{Dynamic profile of blood pressure and cardiac markers}

To determine the cardiovascular implications during COVID-19 progression, the dynamic changes in heart rate, blood pressure, and 5 clinical laboratory parameters, including creatine kinase-MB, $\alpha$ hydroxybutyric dehydrogenase, lactate dehydrogenase, cTnT, and NT-proBNP were tracked in survivors (Figs. 1 and 2). The results of heart rate and blood pressure were recorded every day at the same time. Laboratory parameters were examined once every 3 days. During hospitalization, higher levels of systolic blood pressure were observed in server cases (Fig. 1b). A total of 8 patients from non-severe group and 16 patients from severe group were diagnosed with new onset hypertension (Fig. 1d). The morbidity rate of hypertension was significantly higher in severe cases $(42[76.4 \%]$ vs 27 [44.3\%]; $P=$ 0.0006) (Fig. 1e).

During hospitalization, severe cases exhibited higher levels of cardiac markers (Fig. 2). The prevalence of cardiac complications, including acute cardiac injury and heart failure was significantly higher in severe cases (Table 4). Increases of lactate dehydrogenase and $\alpha$ hydroxybutyric dehydrogenase were also more likely to occur in severe patients (Fig. 2b, c). Myocardial enzymes increased rapidly in the early stage of illness, but gradually decreased with the disease progression. In the end period of the 28-day time frame, abnormal results of cTnT and NT-proBNP were still common in some of the severe patients because of progression of COVID-19 (Fig. 2d, e).

Table 2 Chest Computed Tomographic Images Findings of Patients Infected With COVID-19 on Admission to Hospital

\begin{tabular}{|c|c|c|c|c|}
\hline \multirow[t]{2}{*}{ Abnormalities on chest CT -No./total No. (\%) } & \multicolumn{3}{|l|}{ Disease severity } & \multirow[t]{2}{*}{ PValue } \\
\hline & Total $(N=116)$ & Non-severe $(n=61)$ & Severe $(n=55)$ & \\
\hline Local patchy shadowing & 19/116 (16.4) & $14 / 61(23.0)$ & $5 / 55(9.1)$ & 0.044 \\
\hline Bilateral patchy shadowing & $81 / 116(69.9)$ & $36 / 61(59.0)$ & $47 / 55(85.5)$ & 0.002 \\
\hline Interstitial abnormalities & $4 / 116(3.4)$ & 2/61 (3.3) & 2/55 (3.6) & 0.916 \\
\hline
\end{tabular}


Table 3 Laboratory Findings of Patients Infected With COVID-19 on Admission to Hospital

\begin{tabular}{|c|c|c|c|c|c|}
\hline \multirow[b]{2}{*}{ Laboratory findings } & \multirow[b]{2}{*}{ Normal Range } & \multirow[b]{2}{*}{ Total $(N=116)$} & \multicolumn{2}{|l|}{ Disease severity } & \multirow[b]{2}{*}{$P$ Value } \\
\hline & & & Non-severe $(n=61)$ & Severe $(n=55)$ & \\
\hline White blood cell count, $\times 109 / \mathrm{L}$ & $3.5-9.5$ & $4.9(3.9-6.1)$ & $5.0(3.9-5.7)$ & $4.9(3.8-7.2)$ & 0.3442 \\
\hline$>9.5 \times 109 /$ L, No./total No. (\%) & & $9 / 116(7.8)$ & $1 / 61(1.6)$ & $8 / 55(14.5)$ & 0.0129 \\
\hline$<3.5 \times 109 /$ L, No./total No. (\%) & & $17 / 116(14.7)$ & $9 / 61(14.8)$ & $8 / 55(14.5)$ & 1.0000 \\
\hline Neutrophil count, $\times 109 / L$ & $1.8-6.3$ & $3.1(2.2-4.5)$ & $2.9(2.1-3.8)$ & $3.4(2.5-5.8)$ & 0.0134 \\
\hline Lymphocyte count, $\times 109 / \mathrm{L}$ & $1.1-3.2$ & $1.0(0.7-1.3)$ & $1.2(0.9-1.6)$ & $0.9(0.6-1.1)$ & $<0.0001$ \\
\hline$<1.1 \times 109 /$ L, No./total No. (\%) & & $66 / 116(56.9)$ & 24/61 (39.3) & $42 / 55(76.4)$ & $<0.0001$ \\
\hline Total T cell count, $\times 106 / \mathrm{L}$ & $955-2860$ & 590.5 (361.0-941.8) & $915.0(481.0-1246.0)$ & $448(238.5-644.0)$ & $<0.0001$ \\
\hline$<955 \times 106 /$ L, No./total No. (\%) & & 65/84 (77.4) & $27 / 43(62.8)$ & $38 / 41(92.7)$ & 0.0014 \\
\hline CD4+ T cell count, $\times 106 / L$ & $550-1440$ & $335.0(214.8-493.3)$ & $458.0(275.0-607.0)$ & $262.0(156.5-335.0)$ & 0.0002 \\
\hline$<550 \times 106 /$ L, No./total No. (\%) & & $66 / 84(78.6)$ & 29/43 (67.4) & $37 / 41(90.2)$ & 0.0158 \\
\hline CD8+ T cell count, $\times 106 / L$ & $320-1250$ & $217.0(111.3-359.8)$ & $285.0(144.0-435.0)$ & $157.0(78.5-297.0)$ & 0.0052 \\
\hline$<320 \times 106 /$ L, No./total No. (\%) & & 46/84 (54.8) & 13/43 (30.2) & $33 / 41(80.5)$ & $<0.0001$ \\
\hline Monocyte count, $\times 109 / L$ & $0.1-0.6$ & $0.4(0.3-0.6)$ & $0.4(0.4-0.6)$ & $0.4(0.2-0.6)$ & 0.4886 \\
\hline Platelet count, $\times 109 / \mathrm{L}$ & $125-350$ & $177.0(137.0-230.0)$ & $179.0(145.0-236.0)$ & $161.5(121.8-218.3)$ & 0.1166 \\
\hline Haemoglobinlevel, g/L & $130-175$ & $131.0(118.0-140.0)$ & $129.0(120.0-140.5)$ & $131.0(115.0-140.0)$ & 0.7728 \\
\hline Prothrombin time, $s$ & $10.0-14.0$ & $12.2(11.5-13.0)$ & $11.9(11.5-13.0)$ & $12.4(11.6-13.1)$ & 0.3330 \\
\hline Activated partial thromboplastin time, s & $23.5-39.1$ & $32.3(30.1-34.8)$ & $32.3(30.2-34.6)$ & $32.6(29.8-35.0)$ & 0.8014 \\
\hline D-dimer, ng/mL & $0-243$ & $168.0(92.0-393.5)$ & $122.0(75.0-254.0)$ & $222.0(132.5-529.0)$ & 0.0004 \\
\hline$>243$ ng/mL, No./total No. (\%) & & & $15 / 61(24.6)$ & $25 / 55(45.5)$ & 0.0204 \\
\hline Creatinine, umol/L & $45-110$ & $69.5(56.0-81.0)$ & $68.5(54.3-75.8)$ & $72.0(58.8-86.5)$ & 0.0156 \\
\hline$>110 u m o l / L$, No./total No. (\%) & & $8 / 116(6.9)$ & $0 / 61(0)$ & $8 / 55(14.5)$ & 0.0019 \\
\hline Blood urea nitrogen, $\mathrm{mmol} / \mathrm{L}$ & $2.5-6.3$ & $4.4(3.4-5.8)$ & $3.9(3.2-5.0)$ & $4.9(3.5-7.0)$ & 0.0080 \\
\hline$>6.3 \mathrm{mmol} / \mathrm{L}$, No./total No. (\%) & & 22/116 (19.0) & $5 / 61(8.2)$ & 17/55 (30.9) & 0.0021 \\
\hline Alanine aminotransferase, $\mathrm{U} / \mathrm{L}$ & $9-50$ & $24.0(17.3-37.8)$ & $22.0(15.5-37.5)$ & $27.0(20.0-39.0)$ & 0.1227 \\
\hline$>50.0$ U/L, No./total No. (\%) & & 23/116 (19.8) & $11 / 61(18.0)$ & $12 / 55(21.8)$ & 0.6470 \\
\hline Aspartate aminotransferase, $U / L$ & $15-40$ & $34.5(25.0-52.8)$ & $31.0(22.5-49.5)$ & $38.0(26.0-59.0)$ & 0.0810 \\
\hline$>40.0$ U/L, No./total No. (\%) & & 46/116 (39.7) & $21 / 61(34.2)$ & $25 / 55(45.5)$ & 0.2573 \\
\hline Total bilirubin, umol/L & $4.42-20.52$ & $11.0(8.1-14.1)$ & $11.0(8.3-13.0)$ & $10.5(7.8-16.3)$ & 0.9308 \\
\hline Creatinekinase-MB, U/L & $0-24$ & $17.0(15.0-21.0)$ & $17.0(15.0-22.5)$ & $18.0(15.8-20.0)$ & 0.6960 \\
\hline$>24.0$ U/L, No./total No. (\%) & & 19/116 (16.4) & 12/61 (19.7) & $7 / 55(12.7)$ & 0.4518 \\
\hline Lactate dehydrogenase, U/L & $109-225$ & 245.5 (194.3-319.8) & $225.0(184.0-303.5)$ & $275.0(212.0-378.0)$ & 0.0041 \\
\hline >225.0 U/L, No./total No. (\%) & & $69 / 116(59.5)$ & $30 / 61(49.2)$ & 39/55 (70.9) & 0.0230 \\
\hline Hypersensitive troponin $\mathrm{T}, \mathrm{ng} / \mathrm{mL}$ & $<0.02$ & $0.008(0.005-0.013)$ & $0.007(0.004-0.01)$ & $0.0115(0.006-0.0175)$ & 0.0015 \\
\hline$>0.02$ ng/mL, No./total No. (\%) & & 16/116 (13.8) & $4 / 61(6.6)$ & $12 / 55(21.8)$ & 0.0288 \\
\hline Myoglobin, ng/mL & $28-72$ & $39.4(22.3-92.7)$ & $26.1(21.0-47.8)$ & $54.1(33.4-120.7)$ & 0.0002 \\
\hline$>72.0$ ng/mL, No./total No. (\%) & & $34 / 116(29.3)$ & $12 / 61(19.7)$ & $22 / 55(40.0)$ & 0.0096 \\
\hline NT-proBNP, pg/mL & & $71.5(27.0-363.5)$ & $37.0(19.0-118.8)$ & $233.0(53.3-693.0)$ & $<0.0001$ \\
\hline elevated cases, No./total No. (\%) & & $8 / 116(6.9)$ & $1 / 61(1.6)$ & $7 / 55(12.8)$ & 0.0260 \\
\hline Procalcitonin, ng/mL & $0.00-0.50$ & $0.07(0.03-0.16)$ & $0.05(0.03-0.09)$ & $0.09(0.06-0.23)$ & $<0.0001$ \\
\hline$\geq 0.5 \mathrm{ng} / \mathrm{mL}$, No./total No. (\%) & & $8 / 116(6.9)$ & 2/61 (3.3) & $6 / 55(10.9)$ & 0.1473 \\
\hline $\mathrm{IL}-6, \mathrm{pg} / \mathrm{mL}$ & $0-7.0$ & $18.7(7.7-46.4)$ & $11.8(3.3-27.5)$ & $33.0(13.1-69.2)$ & $<0.0001$ \\
\hline C-reactive protein level, mg/L & $0-10$ & $15.1(9.0-50.9)$ & $10.0(7.0-27.5)$ & $40.0(10.0-8.0)$ & 0.0023 \\
\hline Sodium, mmol/L & $137.0-147.0$ & $138.8(137.1-141.0)$ & $140.0(138.2-141.7)$ & $138.0(134.8-139.3)$ & 0.0001 \\
\hline
\end{tabular}


Table 3 Laboratory Findings of Patients Infected With COVID-19 on Admission to Hospital (Continued)

\begin{tabular}{|c|c|c|c|c|c|}
\hline \multirow[b]{2}{*}{ Laboratory findings } & \multirow[b]{2}{*}{ Normal Range } & \multirow[b]{2}{*}{ Total $(N=116)$} & \multicolumn{2}{|l|}{ Disease severity } & \multirow[b]{2}{*}{ PValue } \\
\hline & & & Non-severe $(n=61)$ & Severe $(n=55)$ & \\
\hline Potassium, mmol/L & $3.5-5.3$ & $4.0(3.5-4.3)$ & $3.9(3.5-4.3)$ & $4.1(3.6-4.3)$ & 0.4548 \\
\hline$>5.3$ mmol/L, No./total No. (\%) & & $1 / 116(0.9)$ & 0/61 (0) & $1 / 55(1.8)$ & 0.4741 \\
\hline$<3.5$ mmol/L, No./total No. (\%) & & 24/116 (20.7) & 12/61 (19.7) & $12 / 55(21.8)$ & 0.8213 \\
\hline Chloride, $\mathrm{mmol} / \mathrm{L}$ & $99.0-110.0$ & $103.3(99.8-105.5)$ & $104.1(101.3-106.2)$ & $102.2(99.2-103.8)$ & 0.0011 \\
\hline Calcium, mmol/L & $2.02-2.6$ & $2.10(2.04-2.21)$ & $2.14(2.09-2.28)$ & $2.07(2.02-2.13)$ & $<0.0001$ \\
\hline Magnesium, mmol/L & $0.6-1.2$ & $0.73(0.68-0.78)$ & $0.76(0.70-0.79)$ & $0.70(0.64-0.76)$ & 0.0025 \\
\hline
\end{tabular}

\section{Discussion}

This retrospective study described the clinical characteristics and cardiovascular implications in hospitalized patients with COVID-19 in Wuhan. By April 13, 2020, of the 116 patients included in this study, 69\% were male, $47 \%$ were severe cases, $90.5 \%$ were discharged, $6.0 \%$ (7) died, and 3.4\% remained hospitalized. Most severe patients were older and had more underlying conditions.
Common symptoms at onset of illness were fever, dry cough, fatigue, dyspnea, and chest discomfort. Local and/or bilateral patchy shadowing was a typical hallmark of CT imaging for COVID-19. Lymphopenia and elevated levels of neutrophil count, C-reactive protein, interleukin 6, D-dimer, creatinine, lactate dehydrogenase, cTnT, and NT-proBNP were more commonly seen in severe cases. During hospitalization, the prevalence of

Table 4 Complications, treatment and outcomes of patients with COVID-19

\begin{tabular}{|c|c|c|c|c|}
\hline \multirow[t]{2}{*}{ Characteristics } & \multirow{2}{*}{$\begin{array}{l}\text { Total }(N= \\
116)\end{array}$} & \multicolumn{2}{|l|}{ Disease severity } & \multirow[t]{2}{*}{ PValue } \\
\hline & & Non-severe $(n=61)$ & Severe $(n=55)$ & \\
\hline \multicolumn{5}{|l|}{ Complications, No./total No. (\%) } \\
\hline Shock & 16/116 (13.8) & $0 / 61(0.0)$ & $16 / 55(29.1)$ & $<0.0001$ \\
\hline Acute cardiac injury & 23/116 (19.8) & $4 / 61(6.6)$ & $19 / 55(34.5)$ & 0.001 \\
\hline Acute respiratory distress syndrome & 20/116 (17.2) & $0 / 61(0.0)$ & 20/55 (36.4) & $<0.0001$ \\
\hline Liver dysfunction & 15/116 (12.9) & 2/61 (3.3) & 13/55 (23.6) & 0.0011 \\
\hline Acute kidney injury & $3 / 116(2.6)$ & $0 / 61(0.0)$ & $3 / 55(5.5)$ & 0.0565 \\
\hline Acute heart failure & 21/116 (18.1) & $3 / 61(4.9)$ & $18 / 55(32.7)$ & 0.0002 \\
\hline \multicolumn{5}{|l|}{ Treatment, No./total No. (\%) } \\
\hline Administration of oseltamivir & $91 / 116(78.4)$ & 46/61 (75.4) & $45 / 55(81.8)$ & 0.4019 \\
\hline Administration of lopinave/litonawe (LPV/r) & 74/116 (63.8) & $42 / 61(68.9)$ & $32 / 55(58.2)$ & 0.2325 \\
\hline Administration of arbidolhydrochloride & 27/116 (23.3) & 15/61 (24.6) & $12 / 55(21.8)$ & 0.7243 \\
\hline Administration of ribavirin injection & $71 / 116(61.2)$ & $37 / 61(60.7)$ & $34 / 55(61.8)$ & 0.8979 \\
\hline Administration of antibiotics & 113/116 (97.4) & $58 / 61(95.1)$ & $55 / 55(100.0)$ & 0.0956 \\
\hline Use of antifungal medications & $12 / 116(10.3)$ & $0 / 61(0.0)$ & $12 / 55(21.8)$ & 0.0001 \\
\hline Administration of systemic corticosteroids & $55 / 116(47.4)$ & $14 / 61(23.0)$ & $41 / 55(74.5)$ & $<0.0001$ \\
\hline Oxygen inhalation & 103/116 (88.8) & $50 / 61(82.0)$ & $53 / 55(96.4)$ & 0.0141 \\
\hline Noninvasive ventilation & 18/116 (15.5) & $0 / 61(0.0)$ & $18 / 55(32.7)$ & $<0.0001$ \\
\hline Invasive mechanical ventilation & 10/116 (8.6) & $0 / 61(0.0)$ & 10/55 (18.2) & 0.0005 \\
\hline Extracorporeal membrane oxygenation & 3/116 (2.6) & $0 / 61(0.0)$ & $3 / 55(5.5)$ & 0.0646 \\
\hline Use of intravenous immunoglobin & $74 / 116(63.8)$ & $25 / 61(41.0)$ & $49 / 55(89.1)$ & $<0.0001$ \\
\hline Use of thymopeptidesinjection & $92 / 116(79.3)$ & 45/61 (73.8) & $47 / 55(85.5)$ & 0.1209 \\
\hline Traditional Chinese medical herbal treatment & 103/116 (88.8) & $55 / 61(90.2)$ & $48 / 55(87.3)$ & 0.2955 \\
\hline \multicolumn{5}{|l|}{ Clinical outcomes, No./total No. (\%) } \\
\hline Discharge from hospital & 109/116 (94.0) & $61 / 61(100.0)$ & $48 / 55(87.3)$ & 0.0040 \\
\hline Death & $7 / 116(6.0)$ & $0 / 61(0.0)$ & $7 / 55(12.7)$ & 0.0040 \\
\hline
\end{tabular}


A

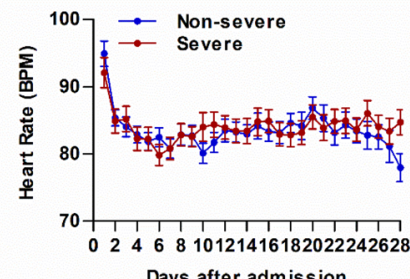

Days after admission

C

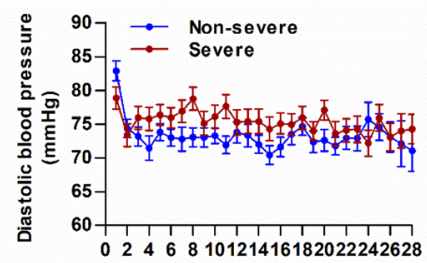

Days after admission

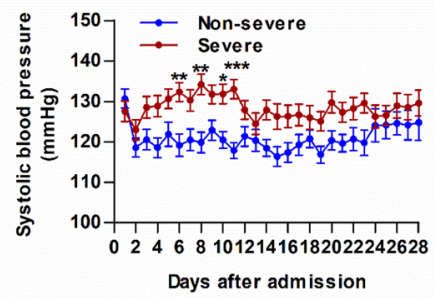

E

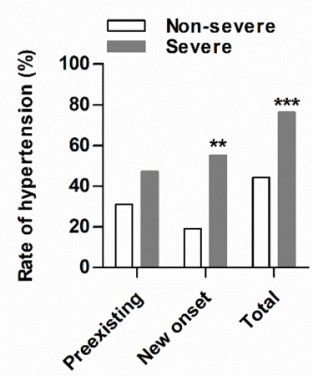

Fig. 1 Dynamic monitoring heart rate and blood pressure of patients hospitalized with COVID-19. During hospitalization, heart rate and blood pressure were recorded every day at the same time by nurses. The dynamic monitoring results of heart rate (a), systolic blood pressure (b), and diastolic blood pressure (c) were compared between non-severe and severe cases. $\mathbf{d}$ The counts of normal blood pressure, preexisting hypertension, and new onset hypertension cases in non-severe and severe groups. e Rate of preexisting hypertension, new onset hypertension, and the total hypertension were compared between non-severe and severe subgroups. ${ }^{*} P<0.05$, ${ }^{* *} P<0.01$, ${ }^{* * *} P<0.001$ for Non-severe vs Severe

new onset hypertension, acute heart injury, and heart failure was significantly higher in severe patients.

In our cohort, 69\% (80) of COVID-19 patient were male. Severe patients were older and had a greater number of comorbid conditions. Evidence from previous studies suggest that older, male patients are the most susceptible to SARS-CoV-2 infection [4, 5, 7, 13], which is supported by our data. It has been confirmed that increased age was associated with death in COVID-19 patients [14], and the coexistence of agedness and comorbidity could lead to an even higher risk of death [13]. Older age has been regarded as an important independent predictor of mortality in COVID-19.

Cardiovascular diseases have a high incidence rate in the middle aged and elderly population [8]. As previously reported [14], we observed that many COVID-19 patients had a comorbidity, with hypertension being the most common (45 [38.8\%]), followed by diabetes (19 [16.4\%]) or coronary heart disease (17 [14.7\%]). The morbidity rates of coronary heart disease and cerebrovascular diseases were significantly higher in the severe group. Thus, older people with comorbidities, such as coronary heart disease and hypertension were thought to be more vulnerable to SARS-CoV-2 and result in more severe outcomes and elevated case-fatality rate [5, $9,15]$. In the present study, 4 of 7 dead patients had preexisting hypertension and coronary heart disease.
Previously, coronary heart disease has also been found to be correlated with acute cardiac events and poor outcomes in influenza and other respiratory viral infections $[16,17]$, Multivariate logistic regression analysis demonstrated that coronary heart disease and heart injury were the independent risk factors for critical disease status in COVID-19 patients [18]. More intense clinical care is in need for COVID-19 patients with cardiac-related chronic diseases.

Incident cardiovascular complications including new or worsening heart failure, new or worsening arrhythmias, or myocardial infarction are common in patients with pneumonia and are associated with increased short-term mortality [19]. Acute pneumonia brings important effects on the status of cardiovascular system irrespective of severities of infection $[16,19]$. Risk factors for cardiac complications after pneumonia include older age, preexisting cardiovascular diseases, and greater severity of pneumonia [16, 19]. An analysis of 112 cardiovascular disease patients with COVID-19 found that, COVID-19 patients combined with cardiovascular disease were associated with a higher risk of mortality [15]. In this study, compared with non-severe patients with COVID-19, severe patients showed abnormalities in numerous cardiac markers. During hospitalization, the morbidity of new onset hypertension, acute heart injury, and heart failure was significantly higher in severe 

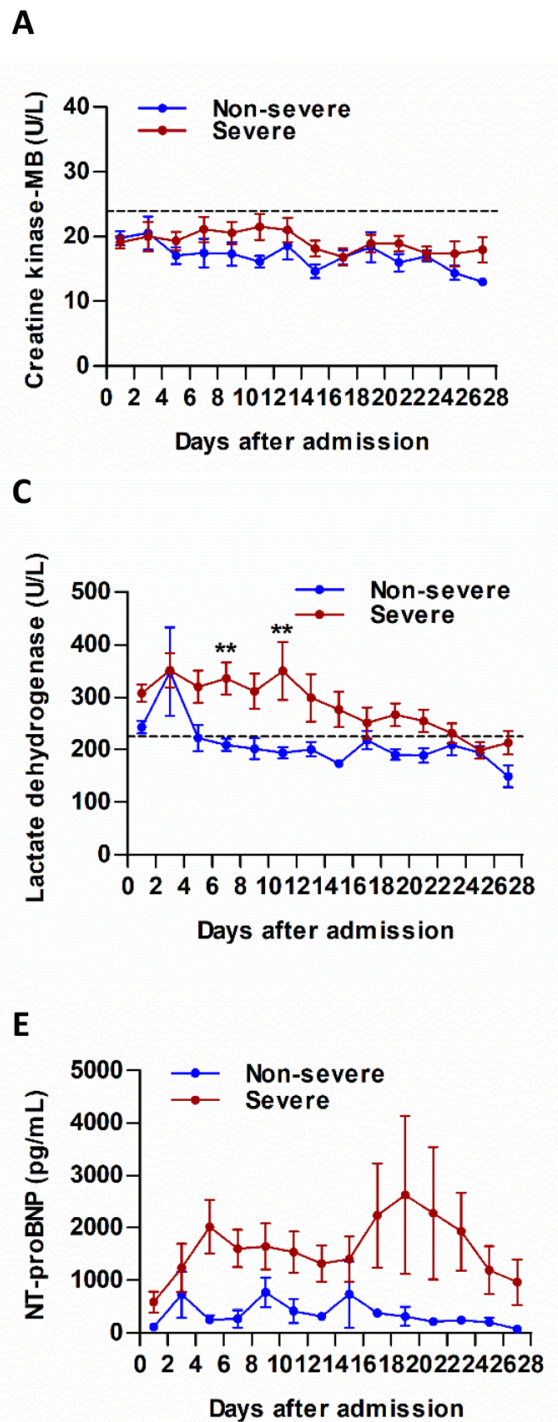
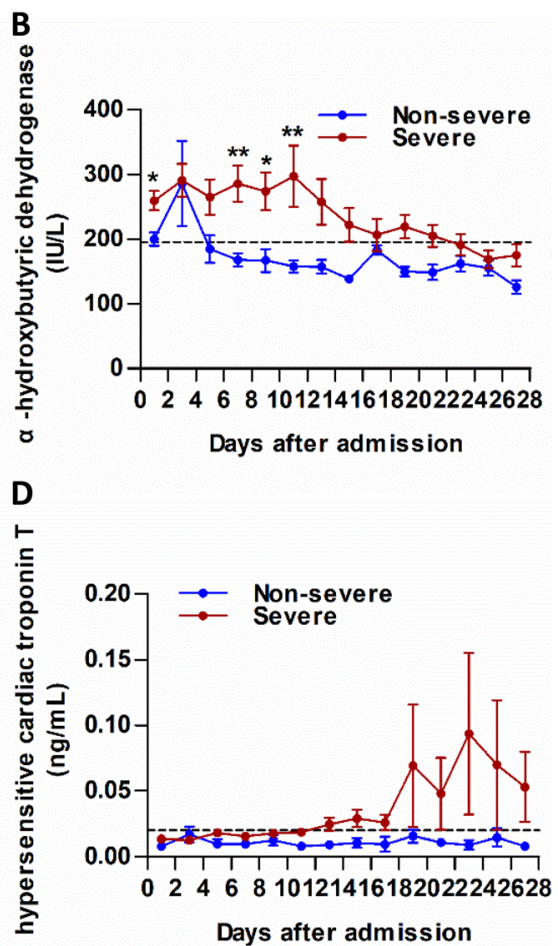

Fig. 2 Temporal changes in laboratory markers in patients hospitalized with COVID-19. Figure shows temporal changes in creatine kinase-MB (a), a-hydroxybutyric dehydrogenase (b), lactate dehydrogenase (c), cTnT (d), and NT-proBNP (e) of non-severe and severe patients every other day after admission. The dotted lines in black show the lower limit of normal for each parameter. ${ }^{*} P<0.05$, ${ }^{* *} P<0.01$ for Non-severe vs Severe

patients. Increased level of myocardial enzymes and cTnT was found in all 3 dead cases. As far as we know, this is the first study that reports the prevalence rate of new onset hypertension was significantly higher in hospitalized severe patients with COVID-19. These findings suggest a higher possibility of cardiovascular complications in severe patients with COVID-19. Outcomes of patients with COVID-19 may be improved by prevention of the development and progression of associated cardiac complications.

Angiotensin-converting enzyme 2 (ACE2) acts as a receptor for SARS-CoV-2 entry into cells and contributes to the pathogenesis of COVID-19 [20]. Meanwhile, ACE2 is widely expressed in myocytes and vascular endothelial cells. At least, these is theoretically a possibility of direct cardiovascular involvement induced by the virus. The result of heart biopsy in a fatal case with COVID-19 showed a few interstitial mononuclear inflammatory infiltration, but no other substantial damage in the heart tissue [21]. However, given that this patient had no clinical manifestations of myocardial injury during the whole course of this disease, it could not be concluded whether myocardium was involved in SARSCoV-2 infection yet. The latest results of autopsy of COVID-19 victims in China demonstrated the existence of RNA and viral particles of SARS-CoV-2 in heart through qRT-PCR-based virus nuclear acid detection, electron microscopy, and immunohistochemical staining [22]. Myocardia displayed cell degeneration, scattered necrosis, interstitial edema, and mild infiltration of 
monocytes, lymphocytes, and/or neutrophils. Multiple postmortem regions showed tunica intima inflammation, thrombosis, anemic infarct [22]. We speculated that the potential pathogenesis of myocardial injury in COVID19 may include several processes, SARS-CoV-2 may directly invade myocytes via ACE2 and cause viral myocarditis; the imbalance between supply and demand in oxygen further results in myocardial injury; and inflammatory cytokines storm. In order to further clarify the etiology of SARS-CoV-2 infection related myocardial injury, it is of great need to obtain pathological evidence from COVID-19 patients showing definite myocardial injury.

Recently, the safety of treatment applying angiotensin converting enzyme inhibitors (ACEI) or angiotensin receptor blockers (ARBs) in relation to COVID-19 has been concerned. An observational study containing 112 patients with cardiovascular diseases infected by COVID-19 reported that there was no significant difference in the proportion of ACEI/ARB medication between the critical group and the general group or between non-survivors and survivors [15]. Currently, it is in lack of any experimental or clinical evidence suggesting adverse or beneficial outcomes with background use of ACEI, ARBs or other RAAS antagonists in COVID-19 or among COVID-19 patients with a history of cardiovascular disease treated with such agents. Statements of ACC and ESC Council on Hypertension do not recommend to discontinue $\mathrm{ACEI} / \mathrm{ARB}$ treatment in the lack of any evidence supporting adverse effect of ACEI and ARB in the context of the pandemic COVID-19 outbreak [23, 24]. Individualized treatment strategies should be approached according to each patient's hemodynamic status and clinical manifestations.

Our study has some limitations. First, only 116 patients with laboratory-confirmed COVID-19 were included. It would be better to include a large population of patients from other centers in Wuhan, and even in other cities in China to get a more comprehensive understanding of COVID-19. Second, not all laboratory tests were dynamically performed in all patients, including the counts of lymphocyte subsets and inflammatory cytokines, therefore their role in the pathogenesis of COVID-19 might be underestimated. Third, due to the retrospective study design, echocardiography and electrocardiograph were only performed in some of the patients. The detailed information of ACEI/ARB medication was incomplete. Therefore, we could not further asses the changes of cardiac structure and function during the progression of COVID-19, and the possible effect of ACEI/ARB on SARS-CoV-2 infection. Last but not least, we were unable to obtain myocardial tissues from deceased COVID-19 patients with heart injury.
The characteristics of myocardial damage should be further demonstrated by pathologists.

\section{Conclusions}

In this single-centered, retrospective, observational study, we found that the infection of SARS-CoV-2 were more likely to occur in older population with cardiovascular comorbidities. Cardiovascular complications, including new onset hypertension and heart injury were common in severe patients with COVID-19. More comprehensive and in-depth researches are in need to unveil the cardiovascular involvement in COVID-19 to further understand the disease.

\section{Abbreviations}

COVID-19: Corona Virus Disease 2019; SARS-CoV-2: 2019 novel coronavirus; ARDS: Acute respiratory distress syndrome; CTnT: Hypersensitive cardiac troponin T; NT-proBNP: N-terminal B-type natriuretic peptide; ACC: American College of Cardiology; ESC: European Society of Cardiology; SEM: Standard error; IQR: Interquartile ranges

\section{Acknowledgements}

Not applicable.

\section{Authors' contributions}

SX was a major contributor in the design of the study, writing the manuscript, and statistical analysis. $L L$ analyzed and interpreted the patient data. $F L, J S, L H, H L, L H, Q J, Z W, W F, Z L, Q L$, and ZC participated in collecting data and interpreting the results. SD designed the study and had full access to all of the data in the study and take responsibility for the integrity of the data and the accuracy of the data analysis. All authors read and approved the final manuscript.

\section{Funding}

This research was supported by the National Natural Science Foundation of China (31600942), Wuhan young and middle-aged medical backbone talent training program, and 2014 Major Projects of PLA Logistics Research Fund (AWS13C003). The funding bodies played no role in the design of the study and collection, analysis, and interpretation of data and in writing the manuscript.

\section{Availability of data and materials}

The datasets used and/or analyzed during the current study are available from the corresponding author on reasonable request.

\section{Ethics approval and consent to participate}

This study was approved by the Ethics Commission of General Hospital of Central Theater Command ([2020]025-1). The ethics committee approved verbal informed consent from all participants or participants' next of kin. Written informed consent was waived by the ethics commission for participants with emerging infectious diseases.

\section{Consent for publication}

Not applicable.

\section{Competing interests}

The authors declare that they have no competing interests.

\section{Author details}

'Department of Cardiology, the General Hospital of Central Theater Command, Wuhan 430000, China. ${ }^{2}$ Department of Integrated Traditional Chinese and Western Internal Medicine, the General Hospital of Central Theater Command, Wuhan 430000, China. ${ }^{3}$ Division of Medical Management, the General Hospital of Central Theater Command, Wuhan 430000, China. 
Received: 14 April 2020 Accepted: 24 September 2020

Published online: 22 October 2020

\section{References}

1. Liu W, Zhang R, Shu R, Yu J, Li H, Long H, Jin S, Li S, Hu Q, Yao F, et al. Study of the relationship between microbiome and colorectal cancer susceptibility using 16SrRNA sequencing. Biomed Res Int. 2020;2020: 7828392.

2. Wu F, Zhao S, Yu B, Chen YM, Wang W, Song ZG, Hu Y, Tao ZW, Tian JH, Pe $Y Y$, et al. A new coronavirus associated with human respiratory disease in China. Nature. 2020.

3. Zhu N, Zhang D, Wang W, Li X, Yang B, Song J, Zhao X, Huang B, Shi W, Lu $R$, et al. A novel coronavirus from patients with pneumonia in China, 2019. N Engl J Med. 2020;382(8):727-33.

4. Guan WJ, Ni ZY, Hu Y, Liang WH, Ou CQ, He JX, Liu L, Shan H, Lei CL, Hui DSC, et al. Clinical characteristics of coronavirus disease 2019 in China. N Engl J Med. 2020.

5. Chen N, Zhou M, Dong X, Qu J, Gong F, Han Y, Qiu Y, Wang J, Liu Y, Wei Y, et al. Epidemiological and clinical characteristics of 99 cases of 2019 novel coronavirus pneumonia in Wuhan, China: a descriptive study. Lancet. 2020; 395(10223):507-13.

6. Huang C, Wang Y, Li X, Ren L, Zhao J, Hu Y, Zhang L, Fan G, Xu J, Gu X, et al. Clinical features of patients infected with 2019 novel coronavirus in Wuhan, China. Lancet. 2020:395(10223):497-506.

7. Wang D, Hu B, Hu C, Zhu F, Liu X, Zhang J, Wang B, Xiang H, Cheng Z, Xiong Y, et al. Clinical characteristics of 138 hospitalized patients with 2019 novel coronavirus-infected pneumonia in Wuhan, China. Jama. 2020;323(11): 1061-9.

8. Joseph P, Leong D, McKee M, Anand SS, Schwalm JD, Teo K, Mente A, Yusuf S. Reducing the global burden of cardiovascular disease, part 1: the epidemiology and risk factors. Circ Res. 2017;121(6):677-94.

9. Wu Z, McGoogan JM. Characteristics of and important lessons from the coronavirus disease 2019 (COVID-19) outbreak in China: summary of a report of 72314 cases from the Chinese Center for Disease Control and Prevention. Jama. 2020

10. WHO. Clinical management of severe acute respiratory infection when novel coronavirus (nCov) infection is suspected. 2020. https://www.who.int/ publications-detail/clinical-management-of-severe-acute-respiratoryinfection-when-novel-coronavirus-(ncov)-infection-is-suspected.

11. Kellum JA, Lameire N, Group KAGW. Diagnosis, evaluation, and management of acute kidney injury: a KDIGO summary (part 1). Crit Care. 2013;17(1):204.

12. Ponikowski P, Voors AA, Anker SD, Bueno H, Cleland JGF, Coats AJS, Falk V, González-Juanatey JR, Harjola V-P, Jankowska EA, et al. 2016 ESC guidelines for the diagnosis and treatment of acute and chronic heart failure: the task force for the diagnosis and treatment of acute and chronic heart failure of the European Society of Cardiology (ESC) developed with the special contribution of the Heart Failure Association (HFA) of the ESC. Eur Heart J. 2016:37(27):2129-200.

13. Yang $X, Y u Y, X u J$, Shu $H$, Xia J, Liu H, Wu Y, Zhang L, Yu Z, Fang $M$, et al. Clinical course and outcomes of critically ill patients with SARS-CoV-2 pneumonia in Wuhan, China: a single-centered, retrospective, observational study. Lancet Respir Med. 2020.

14. Zhou F, Yu T, Du R, Fan G, Liu Y, Liu Z, Xiang J, Wang Y, Song B, Gu X, et al. Clinical course and risk factors for mortality of adult inpatients with COVID19 in Wuhan, China: a retrospective cohort study. Lancet. 2020.

15. Peng YD, Meng K, Guan HQ, Leng L, Zhu RR, Wang BY, He MA, Cheng LX, Huang K, Zeng QT. Clinical characteristics and outcomes of 112 cardiovascular disease patients infected by 2019-nCoV. Zhonghua Xin Xue Guan Bing Za Zhi. 2020;48(0):E004.

16. Corrales-Medina VF, Musher DM, Shachkina S, Chirinos JA. Acute pneumonia and the cardiovascular system. Lancet. 2013;381(9865):496-505.

17. Udell JA, Zawi R Fau - Bhatt DL, Bhatt DI Fau - Keshtkar-Jahromi M, Keshtkar-Jahromi M Fau - Gaughran F, Gaughran F Fau - Phrommintikul A, Phrommintikul A Fau - Ciszewski A, Ciszewski A Fau - Vakili H, Vakili H Fau Hoffman EB, Hoffman Eb Fau - Farkouh ME, Farkouh Me Fau - Cannon CP et al: Association between influenza vaccination and cardiovascular outcomes in high-risk patients: a meta-analysis. Jama 2013, 310(16):17111720 .

18. Chen C, Chen C, Yan JT, Zhou N, Zhao JP, Wang DW. Analysis of myocardial injury in patients with COVID-19 and association between concomitant cardiovascular diseases and severity of COVID-19. Zhonghua Xin Xue Guan Bing Za Zhi. 2020:48(0):E008.

19. Corrales-Medina VF, Musher DM, Wells GA, Chirinos JA, Chen L, Fine MJ. Cardiac complications in patients with community-acquired pneumonia: incidence, timing, risk factors, and association with short-term mortality. Circulation. 2012;125(6):773-81.

20. Xu X, Chen P, Wang J, Feng J, Zhou H, Li X, Zhong W, Hao P. Evolution of the novel coronavirus from the ongoing Wuhan outbreak and modeling of its spike protein for risk of human transmission. Sci China Life Sci. 2020.

21. Xu Z, Shi L, Wang Y, Zhang J, Huang L, Zhang C, Liu S, Zhao P, Liu H, Zhu L, et al. Pathological findings of COVID-19 associated with acute respiratory distress syndrome. Lancet Respir Med. 2020.

22. Bian X-W, Team tC-P. Autopsy of COVID-19 victims in China. Natl Sci Rev. 2020:nwaa123 https://doi.org/10.1093/nsr/nwaa123.

23. American College of Cardiology. ACC Clinical Bulletin: Cardiac Implications of Novel Wuhan Coronavirus (COVID-19). 2020.

24. ESC Council on Hypertension: Position Statement of the ESC Council on Hypertension on ACE-Inhibitors and Angiotensin Receptor Blockers. 2020.

\section{Publisher's Note}

Springer Nature remains neutral with regard to jurisdictional claims in published maps and institutional affiliations.
Ready to submit your research? Choose BMC and benefit from:

- fast, convenient online submission

- thorough peer review by experienced researchers in your field

- rapid publication on acceptance

- support for research data, including large and complex data types

- gold Open Access which fosters wider collaboration and increased citations

- maximum visibility for your research: over $100 \mathrm{M}$ website views per year

At $\mathrm{BMC}$, research is always in progress.

Learn more biomedcentral.com/submissions 